Signal \& Image Processing : An International Journal (SIPIJ) Vol.8, No.2, April 2017

\title{
APPLYING EDGE INFORMATION IN YCbCr COLOR SPACE ON THE IMAGE WATERMARKING
}

\author{
Mansur Jaba $^{1}$, Mosbah Elsghair ${ }^{2}$, Najib Tanish ${ }^{1}$ and Abdusalam Aburgiga ${ }^{2}$ \\ ${ }^{1}$ Alpha University, Serbia and \\ ${ }^{2}$ John Naisbitt University, Serbia
}

\begin{abstract}
Copyright protection has currently become a difficult domain in reality situation. an honest quality watermarking scheme might to have high sensory activity transparency, and may even be robust enough against potential attacks. This paper tends to propose the special domain based mostly watermarking scheme for color pictures. This scheme uses the Sobel and canny edge detection strategies to work out edge data of the luminance and chrominance elements of the colour image. The edge detection strategies are used to verify the embedding capability of every color element. The massive capacities of watermark bits are embedded into an element of enormous edge information. The strength of the projected scheme is analyzed considering differing kinds of image process attacks, like Blurring and adding noise.
\end{abstract}

\section{KEYWORDS}

Edge, Watermarking, RGB, YCbCr, Sobel Operator, Canny's Edge Detection.

\section{INTRODUCTION}

The color space illustration has competed a significant role in committal to writing, compression, transmission, pattern recognition and digital transmission applications. Moreover, color has become a lot of recently a serious element in watermarking applications of transmission contents [1]. The image watermarking schemes are accustomed defend the copy rights of digital images [2]. Many schemes are projected to implant the watermark in gray-scale images [3]. But, only a few schemes are designed for color images. In color pictures the embedding of watermark could also be done either in special domain or frequency domain [4]. The special domain schemes embed the watermark by directly modifying the colour intensity values of the duvet image and these schemes are less complicated in computation [5]. On the opposite hand, frequency domain schemes [6], [7] the watermark is embedding by modulating the frequency coefficients of the image transformation. The foremost wide used frequency remodeled domains used for watermarking are discrete cosine transform (DCT) [8], discrete Fourier transform (DFT) and discrete wavelet Transformation(DWT) [9], [10], [11]. The foremost wide used color representations are RGB, YUV, YCbCr, HSV and CIELAB [10]. The YUV illustration is employed in watermarking of color data in TV systems, because the $\mathrm{YCbCr}$ is employed in digital watermarking of color data in video and still-image. The RGB color space illustration has the foremost correlative elements, whereas the $\mathrm{YCbCr}$ color elements are the smallest amount 
correlated elements. The forward and backward transforms between RGB and YCbCr color areas are linear. The correlated RGB elements do not seem to be appropriate to embed the watermark. In RGB color area the perceived color quality of a picture depends on all elements. Thus, embedding watermark bits into one element independently of the opposite RGB elements is not the most effective alternative. On the opposite hand the $\mathrm{YCbCr}$ permits to extract uncorrelated elements and it favor the separation of the achromatic half from the chromatic elements of the colour image. To realize high robustness and enormous embedding capability, the projected scheme uses the smallest amount correlative $\mathrm{YCbCr}$ elements of the colour image. The colour image is pictured by $\mathrm{Y}, \mathrm{Cb}$ and $\mathrm{Cr}$ elements. From these three parts, the modification within the intensity of chrominance parts is that the most sensitive to human eyes whereas for brightness level parts are least sensitive [12]. Thus, the projected scheme uses the brightness level part for embedding the watermark. The paper is organized as follows: In Section two the edge detection strategies are explained. The projected schemes with embedding and extraction algorithmic rule are delineated in Section three. The results and discussions are given in Section four. the consequences of the image process attacks are analyzed in Section five. The paper is complete in Section six. Fig 1 is shown that representation of a natural.

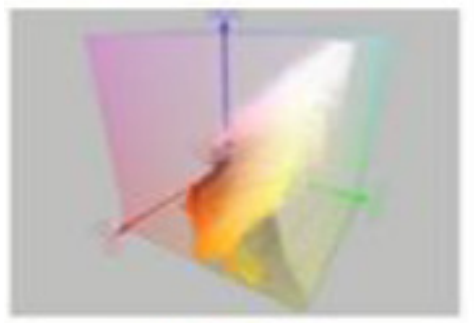

(a) RGB

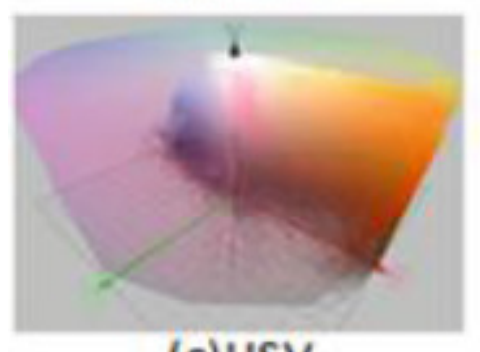

(c)HSV

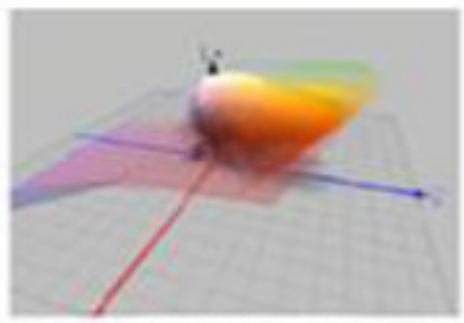

(b) $I^{*} a^{*} b$

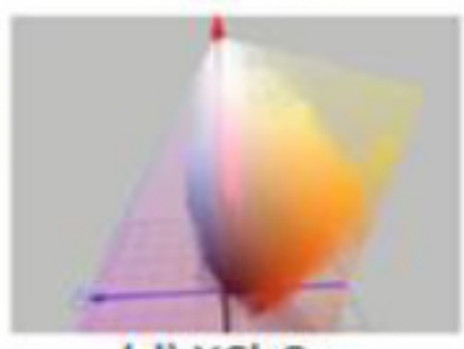

(d) $\mathrm{YCbCr}$

Figure 1. Representation of a natural image with several color: RGB, L*a*b, HSV and YCbCr

\section{EDGE DETECTION TECHNIQUE}

The edge detection is that the method of characteristic and locating the sharp discontinuities in a picture [13]. The discontinuities square measure abrupt changes in pixel intensity that characterize boundaries of objects in a picture. There square measure several edge detection operators obtainable, every operator is sensitive to sure sorts of edges. The variable factors that square measure concerned in choice of an edge detection algorithmic rule include. 
Signal \& Image Processing : An International Journal (SIPIJ) Vol.8, No.2, April 2017

\subsection{Edge Orientation:}

The pure mathematics of the algorithmic rule determines a characteristic direction within which it may most sensitive to edges Operators may be optimized to seem for horizontal, vertical, or diagonal edges.

\subsection{Noise Environment:}

Edge detection is tough in noisy pictures, since each the noise and therefore the edges contain high frequency. This makes an attempt to decrease the noise lead to blurred and distorted edges. Operators used on noisy pictures square measure larger in scope, they may average enough to eliminate the localized noisy pixels.

\subsection{Edge Structure :}

Not all edges involve a step amendment in intensity. Effects of refraction or poor focus might lead to objects with boundaries outlined by a gradual amendment in intensity. The algorithmic rule has to be chosen must be tuned in to amendment.

\subsection{Sobel Operator:}

This operator consists of a $3 * 3$ convolution kernels. The kernels square measure organized such as one kernel is alternative kernel turned by 90 . These kernels square measure designed to horizontally relative to the pixel grid and reply maximally to edges running vertically. The kernels may be applied on an individual basis to the input image to supply separate measurements of the gradient element in every orientation. These may be combined together to search out absolutely the magnitude of the gradient at every purpose and therefore the orientation of gradient.

\subsection{Canny's Edge Detection:}

The canny edge detection algorithmic rule is understood to several because the optimal edge detector. The canny's algorithmic rule followed an inventory of criteria to enhance current strategies of edge detection. the first and most evident is low error rate. it may necessary that edges occurring in images must not be incomprehensible and there are going to be no responses to non-edges. The second criterion is that the edge points be localized. In alternative words, the space between the edge pixels as found by the detector the actual edge is to be token. A third criterion is to possess just one response to one edge. Supported these criteria, the canny edge detector initial smooth the image to eliminate the noise.

\section{PROPOSED SCHEME}

The projected scheme uses the colour image I of size $\mathrm{m}^{*} \mathrm{n}$ because the cover image and therefore the watermark $\mathrm{W}$ of size $\mathrm{P}$ bits. the colour image is transformed into $\mathrm{R}, \mathrm{G}$ and $\mathrm{B}$ elements of size $\mathrm{m} \mathrm{n} * \mathrm{R}, \mathrm{G}$ and $\mathrm{B}$ element square measure transformed into $\mathrm{Y}, \mathrm{Cb}$ and $\mathrm{Cr}$ elements employment following equations. 
Signal \& Image Processing : An International Journal (SIPIJ) Vol.8, No.2, April 2017

$$
\begin{aligned}
& Y=0.299 \times R+0.587 \times G+0.114 \times B \\
& C b=0.596 \times R-0.275 \times G-0.321 \times B \\
& C r=0.212 \times R-0.523 \times G-0.311 \times B
\end{aligned}
$$

Since, the human eyes square measure less sensitive to alter within the intensity of the Y element, the watermark bits area unit LSB substituted into the Y element. On Y, Cb and $\mathrm{Cr}$ elements the Sobel and canny edge detection strategies area unit applied to work out the high edge elements. The pixels of the high edge element area unit LSB substituted with watermark bits. The embedded $\mathrm{Y}, \mathrm{C} \mathrm{b}$ and $\mathrm{C} \mathrm{r}$ elements area unit transformed into RGB elements using following equations.

$$
\begin{aligned}
& R^{0}=Y^{0}+0.956 \times C b+0.620 \times C r(4) \\
& G^{0}=Y^{0}-0.272 \times C b+0.647 \times C r(5) \\
& B^{0}=Y^{0}-1.108 \times C b+1.705 \times C r \quad(6)
\end{aligned}
$$

From the embedded R'G'B' elements are combined to realize watermarked color image. Fig. 2: shows the flow chart of the embedding algorithmic rule.

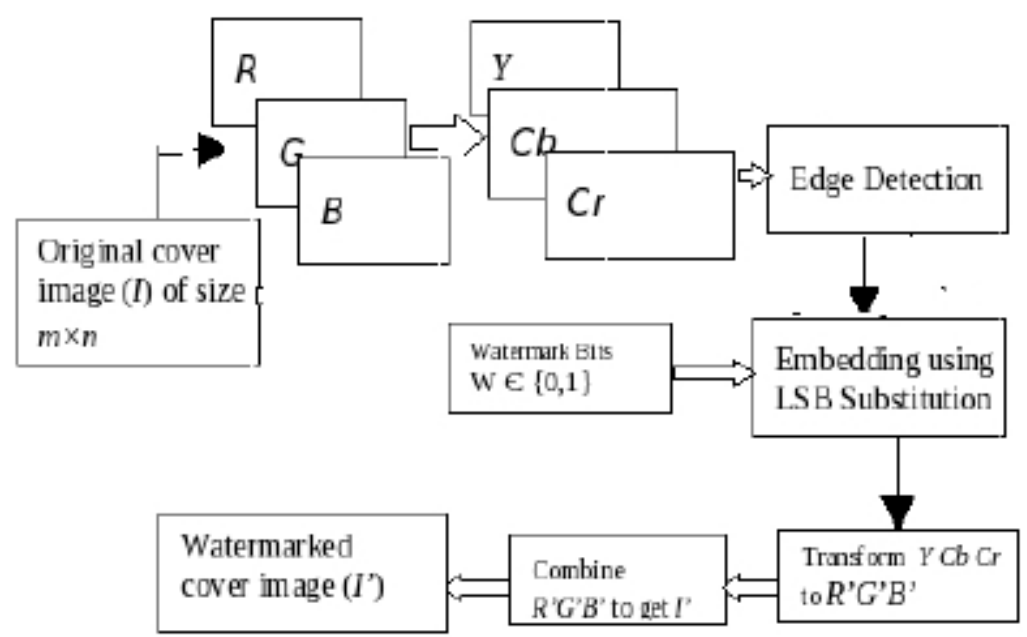

Figure 2: Flow diagram of embedding algorithm

\section{Algorithm:1. Embedding:}

Input: A Color original image I of size $m^{*} n$, watermark W of size P bits, wherever $\mathrm{W} €\{0,1\}$.

Output: Watermarked image I zero of size m*n.

1) Separate R, G and B components of size $m * n$ from color image I.

2) Transform R, G and B elements in to $\mathrm{Y}, \mathrm{Cb}, \mathrm{Cr}$ elements using (1), (2) and (3).

3) Verify Edge info from Y, Cb, Cr elements using Sobel/Canny methodolog. 
Signal \& Image Processing : An International Journal (SIPIJ) Vol.8, No.2, April 2017

4) Choose the element from $\mathrm{Y}, \mathrm{Cb}, \mathrm{Cr}$ that is having massive edge information i.e.Y.

5) Embed watermark bit $\mathrm{W} €\{0,1\}$.

6) Transform Y zero, Cb, Cr into R', G', B' elements using (4), (5) and (6).

7) Combine R', G', B' elements to come up with watermarked color image I.

This table 1 is illustrated to use the algorithm watermark embedding with original image.

Table 1: algorithm Watermark embedding

\begin{tabular}{|l|l|l|}
\hline Original Image & Watermark & Watermarked \\
\hline & & \\
\hline & & \\
\hline
\end{tabular}

\section{Algorithm: 2. Extraction}

Input: A Color watermarked picture I' of size $\mathrm{m}^{*} \mathrm{n}$.

Output: Original image I of size $m * n$.

1) Separate R, G ' and B ' elements of size $m$ * $n$ from watermarked color image I ' .

2) Rework R ', G 'and B' elements in to Y', Cb' Cr' elements using (1), (2) and (3).

3) Verify Edge data from every element using Sobel/canny technique.

4) choose the element from $\mathrm{Y}^{\prime}, \mathrm{C} \mathrm{b}^{\prime}, \mathrm{C} \mathrm{r}^{\prime}$ that is having massive edge data i.e., $\mathrm{Y}^{\prime}$.

5) Extract watermark bit $\mathrm{W} \in\{0,1\}$ from the LSB of the pixels. 
Signal \& Image Processing : An International Journal (SIPIJ) Vol.8, No.2, April 2017

The embedding and therefore the extraction algorithms are given in algorithmic rule 1: and algorithmic rule 2: respectively. Consequence, this is figure 3 can show extraction algorithm flow diagram.

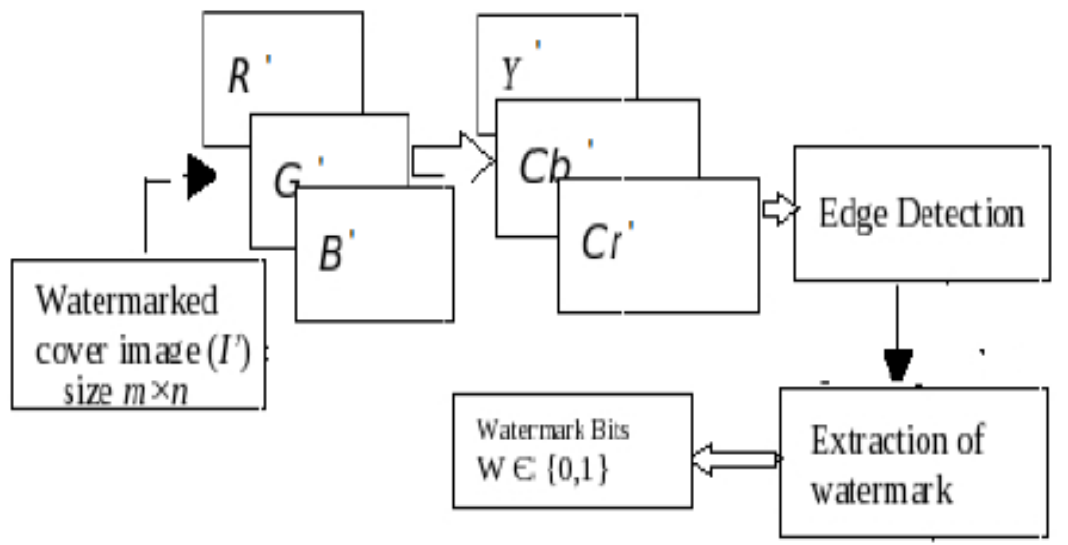

Figure 3: Flow diagram of extraction algorithm

In extraction algorithmic rule, the proposal uses the watermarked color image I zero of size $m^{*} \mathrm{n}$. The watermarked image is reworked into R', G', B' elements of size m*n. The R', G', B' color elements are reworked into $\mathrm{Y}^{\prime}$ zero, $\mathrm{Cb}^{\prime}$ and $\mathrm{Cr}^{\prime}$ using (1), (2) and (3). the sting detection strategies are applied on these elements to work out element that is having massive edge data. The element that has massive edge is taken into account within the extraction of the watermark bits and this Table 2: This table is illustrated the algorithm watermark extraction algorithm.

Table2: algorithm Watermark extraction

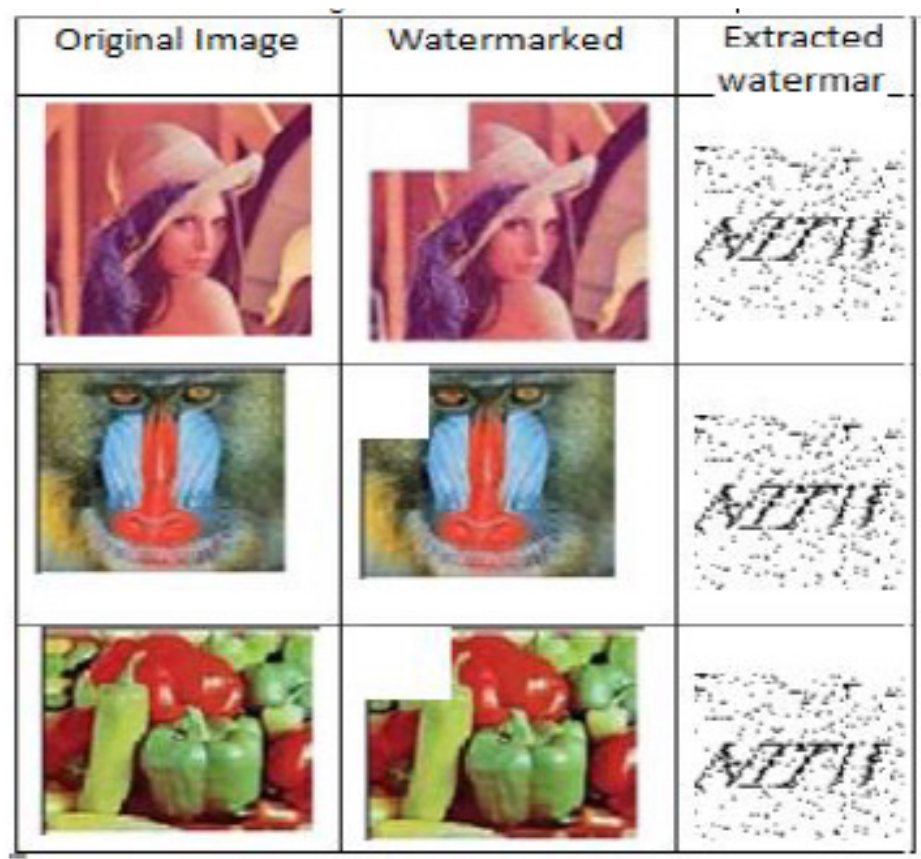




\section{RESULTS AND DISCUSSION}

The series of experiments square measure conducted to analyze the impact of embedding and extraction algorithmic rule on the color pictures. In these experiments, the colour pictures of Lena, Blue hills, Sunset and Water Lilies of different sizes square measure thought of because the cover images. The watermark $\mathrm{W} €\{0,1\}$, is generated exploitation pseudo random binary generator such as in figure 4 and figure 5 . The experiments square measure conducted estimate embedding capability of the planned scheme using completely different edge detection strategies. The parameters like Mean Quadrangle Error (MSE), Peak Signal to Noise magnitude relation (PSNR), Normalized Correlation (NC) and normal Correlation (SC) will not measure the performance of those.

(a) Blue Hilles

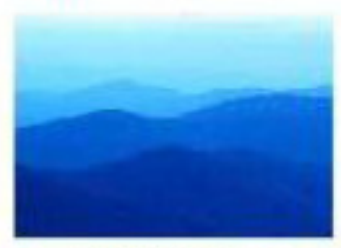

(c) Sunset

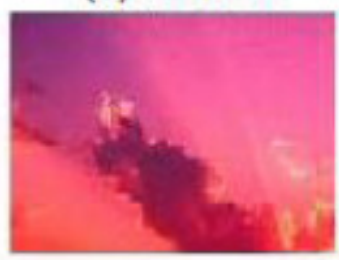

Figure 4: The Original Color Cover Image

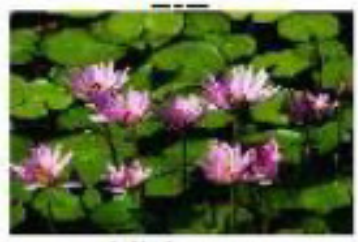

(d) Lena (a) Blue Hilles

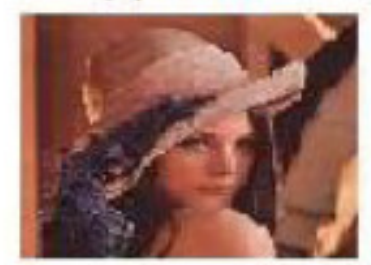

(c) Sunset

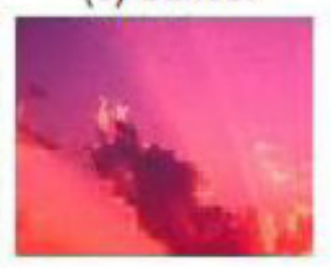

Figure 5: Watermarked Images
(b)Water Liles

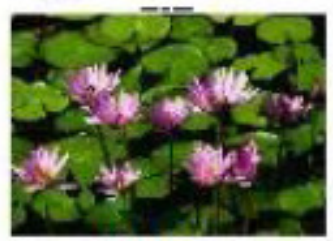

(d) Lena

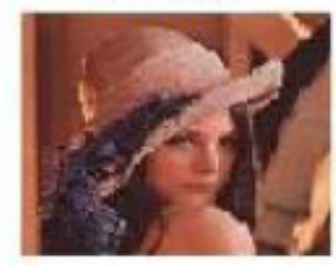

The parameters like Mean Quadrangle Error (MSE), Peak Signal to Noise magnitude relation (PSNR), Normalized Correlation (NC) and normal Correlation (SC) are wont to measure the performance of embedding and extraction algorithms.

\section{1) Structured Correlation (SC):}

It measures however the pixel values of original image square measure correlated with the pixel values of changed image. There is no distortion in changed image, then SC can be :

$$
S C=\frac{\sum_{i=1}^{M} \sum_{j=1}^{N}\left(I[i, j]-I^{\prime}\right)\left(J[i, j]-J^{\prime}\right)}{\sqrt{\sum_{i=1}^{M} \sum_{j=1}^{N}\left(I[i, j]-I^{\prime}\right) \sqrt{\sum_{i=1}^{M} \sum_{j-1}^{N}\left(J[i, j]-J^{\prime}\right)}}}
$$

\section{2) Normalized Correlation (NC):}

It measures the similarity illustration between the initial image and therefore the changed image. 
Signal \& Image Processing : An International Journal (SIPIJ) Vol.8, No.2, April 2017

$$
N C=\frac{\sum_{i=1}^{M} \sum_{j=1}^{N}\left(I[i, j] I^{\prime}[i, j]\right)}{\sum_{i=1}^{M} \sum_{j=1}^{N}(I[i, j])^{2}}
$$

where, $I(i, j)$ is original image and that $I^{\prime}(i, j)$ is changed image, $M$ is height of image and $N$ is image dimension.

\section{3) Mean square. Error (MSE):}

It measures the typical of the square of the error. The error is that the quantity by that the pixel worth of original image differs from the pixel worth of changed image.

$$
M S E=\frac{\sum_{\mathbf{s}\left\{R, G, E_{i}\right.} \sum_{i=1}^{M} \sum_{j=1}^{N}\left(I[i, j]_{k}-I^{\prime}[i, j]_{k}\right)^{2}}{3 M N}
$$

where, $\mathrm{M}$ and $\mathrm{N}$ are the peak and dimension of image severally. $f(i, j)$ is that the $(i, j)$ the pixel worth of the original image and $f(i, j)$ is the $(i, j)$ th pixel worth of changed image.

\section{4) Peak Signal to Noise magnitude relation (PSNR):}

It might the magnitude relation between the most possible power of a signal and therefore the power of corrupting noise that affects the fidelity of its illustration. PSNR is typically expressed in terms of the exponent sound unit. PSNR is given by.

$$
P S N R=\frac{10 \log \left(2^{n}-1\right)^{2}}{M S E}
$$

The calculable values of MSE and PSNR between the initial and watermarked images exploitation Sobel edge detection methodology. From the results analysis of it absolutely was determined that the canny edge detection methodology might help to determine the additional edge information obtainable in an image. Exploitation Canny edge detection is able to embed sizable amount of watermark bits into original images as such in table 3

Table 3: PSNR and MSE between Watermarked and Original Image for slob

\begin{tabular}{|c|c|c|c|}
\hline Image Name & Size of image & MSE & PSNR $(\mathbf{d B})$ \\
\hline Lena & $225 \times 225$ & 0.0060 & 26.0512 \\
\hline Sunset & $800 \times 600$ & 0.0021 & 29.7994 \\
\hline Blue Hills & $225 \times 225$ & $5.3125 \mathrm{e}-004$ & 35.8200 \\
\hline Water Lilies & $800 \times 600$ & $5.3125 \mathrm{e}-004$ & 35.8200 \\
\hline
\end{tabular}

The calculable values of MSE and PSNR between the initial and Watermarked images exploitation canny edge detection methodology such as in table 4. 
Signal \& Image Processing : An International Journal (SIPIJ) Vol.8, No.2, April 2017

Table 4: PSNR and MSE between Watermarked and Original Image for canny.

\begin{tabular}{|c|c|c|c|}
\hline Image Name & Size of image & MSE & PSNR (dB) \\
\hline Lena & $225 \times 225$ & 0.0070 & 25.0512 \\
\hline Sunset & $800 \times 600$ & 0.0051 & 27.7994 \\
\hline Blue Hills & $225 \times 225$ & 0.0031 & 22.8200 \\
\hline Water Lilies & $800 \times 600$ & 0.004 & 32.8200 \\
\hline
\end{tabular}

The NC and SC between extracted and cover watermark bits for various cover images. The experimental results show that the NC and SC for all images square measure adequate to 1 that shows that the extracted watermark bits are correlative to the initial watermark bits as the shown in table 5 .

Table 5: The NC and SC between the extracted and original watermark

\begin{tabular}{|c|c|c|c|c|}
\hline $\begin{array}{c}\text { Propertie } \\
\text { e }\end{array}$ & Lena & Sunset & Blue Hills & Water Lilies \\
\hline NC & 1.00 & 1.00 & 1.00 & 1.00 \\
\hline SC & 1.00 & 1.00 & 1.00 & 1.00 \\
\hline
\end{tabular}

\section{EFFECT OF ATTACKS}

In this section, we have a tendency to justify briefly the impact of image process attacks against the watermarked picture. The attacked watermarked image is estimated and tested so as to validate the projected scheme. when extracting the watermark from the attacked watermarked picture. The Normalized or relation (NC) is determined between the extracted watermark and bits of original image. The subsequent section describes the impact of Gaussian blurring and adding Gaussian noise on watermarked.

\subsection{Adding Gaussian Noise:}

One of the un-intentional attacks which might occur on the quilt pictures is addition of noise. A series of experiments square measure conducted to research the impact of adding Gaussian noise to the watermarked pictures. The noise density is varied from five pixels to twenty-five pixels and noise is extra to the watermarked pictures. From these watermarked pictures the watermark is extracted and compared with original watermark in terms of NC. The result of adding Gaussian noise on the set of canopy pictures, wherever the PSNR and NC of extracted watermarks with original watermark shows that the watermark square measure less distorted as shown in table 6 .

Table 6: The effect of adding Gaussian noise on watermarked and original watermark

\begin{tabular}{|c|c|c|c|c|}
\hline Noise Density & Lena & Sunset & Blae Hills & Water Lilies \\
\hline 3 & 1 & 0.99 & 0.91 & 0.9 \\
\hline 10 & 0.98 & 0.9 & 0.88 & 0.87 \\
\hline 15 & 0.9 & 0.88 & 0.8 & 0.81 \\
\hline 20 & 0.88 & 0.8 & 0.8 & 0.8 \\
\hline 25 & 0.87 & 0.8 & 0.8 & 0.18 \\
\hline
\end{tabular}


Signal \& Image Processing : An International Journal (SIPIJ) Vol.8, No.2, April 2017

\subsection{Effect of Gaussian Blurring:}

The Blurring is applied on the watermarked color original pictures. within the analysis of attacks, it tends to applied Gaussian blurring with radius of various from five to twenty-five pixels. The parallelogram blurring is applied by variable the blurring size from five pixels to twenty-five pixels to the watermarked pictures. The impact of Gaussian blurring on set of pictures as in table 7.

Table 7: Gaussian blurring impact on watermark images in terms of NC between the extracted and original watermark

\begin{tabular}{|c|c|c|c|c|}
\hline Blurring width & Lena & Sunset & Blae Hills & Water Lilies \\
\hline 5 & 1 & 1.00 & 0.59 & 0.90 \\
\hline 10 & 0.90 & 0.95 & 0.91 & 0.90 \\
\hline 15 & 0.90 & 0.89 & 0.50 & 0.80 \\
\hline 20 & 0.85 & 0.84 & 0.88 & 0.81 \\
\hline 25 & 0.82 & 0.81 & 0.81 & 0.81 \\
\hline
\end{tabular}

\section{CONCLUSiON}

It has got projected an abstraction domain primarily based blind watermarking theme. The $\mathrm{YCbCr}$ elements square measure thought of to implant the watermark. Since, the Y element defines massive edge data the watermark is implant into the $\mathrm{Y}$ element. The watermark is embedded into $\mathrm{Y}$ element and it might terribly tough to take off or destroy the watermark. The watermarking scheme is analyzed by considering numerous varieties of image process attacks and also the scheme was found robust to numerous varieties of image process attacks.

\section{REFERENCES}

[1] I.J. Cox, M.L. Miller, J. A. Bloom, J. Fridrich, T. Kalker, "Digital Watermarking and Steganography”, Second edition, Morgan Kaufmann Publishers, 2008.

[2] Nagaraj V. Dharwadkar, B. B. Amberker,"Secure Watermarking Scheme for Color Image Using Intensity of Pixel and LSB Substitution", Journal of Computing, vol.1, issue 1, pp 1-6, 2009.

[3] A.A. Reddy, B.N. Chatterjii, "A new wavelet based logo-watermarking scheme, Pattern Recognition Letters”, vol. 26, pp. 1019-1027, 2005.

[4] P. Vidyasagar, H. Song and C. Elizabeth., "A survey of digital image watermarking techniques", IEEE 3rd 132 International Conference on Industrial Informatics, Frontier Technologies for the Future of Industry and Business, pp.709-716, 2005.

[5] Nagaraj V. Dharwadkar, B. B. Amberker, "Watermarking Scheme for Color Images using Wavelet Transform based Texture Properties and Secret Sharing", International Journal of Signal Processing, vol.6, issue 2, pp.93-100, 2010.

[6] P. Meerwald, A. Uhl, "A survey on wavelet domain watermarking algorithms", Proceedings of SPIE, Electronic Imaging, Security and Watermarking of Multimedia Contents III, vol. 4314, pp. 505-516, 2001.

[7] D. Kundur, D. Hatzinakos, "Towards robust logo watermarking using multi-resolution image fusion", IEEE Transactions on Multimedia, vol.6, pp.185-197, 2004. 
Signal \& Image Processing : An International Journal (SIPIJ) Vol.8, No.2, April 2017

[8] Wai C. Chu,"DCT-Based Image Watermarking Using Subsampling", IEEE Transactions on Multimedia, vol. 5, issue 1, pp. 34-38, 2003

[9] Min-Jen Tsai and Hsiao-Ying Hung, "DCT and DWT-based Image Watermarking by Using Subsampling", Proceedings of 24th International Conference on Distributed Computing Systems Workshops, pp. 1-6, 2004.

[10] Nagaraj V. Dharwadkar, B.B. Amberker, "An Efficient Non-blind Watermarking Scheme for Color Images using Discrete Wavelet Transformation", vol.2, issue 3, pp.60-66, 2010.

[11] Nagaraj V. Dharwadkar, B.B. Amberker, "An Efficient and Secured Non Blind Watermarking Scheme for. Color Images Using DWT and Arnold Transform", International Journal of Computing, vol.9, issue 2, pp. 183-191, 2010.

[12] M. Kutter, F. Jordan, and F. Bossen, "Digital signature of color images using amplitude modulation", in Proc. SPZE Int. Conf: Storage and Retrieval for Zmage and Video Database, vol. 3022, pp. 518526, 1997.

[13] Gonzalez, Woods, and Eddins, "Digital Image Processing Using MATLAB", Gatesmark Publishing, 2009. 